\title{
DISRUPTION OF PROPRIOCEPTIVE INFORMATION DURING ELECTRICAL STIMULATION OF THE CUTANEOUS AFFERENTS
}

Natalija Katic ${ }^{1,2 *}$, Josep-Maria Balaguer ${ }^{3 *}$, Oleg Gorskii ${ }^{4,5,6}$, Natalia Pavlova ${ }^{4}$, Dzhina Karalogly $^{7}$, Dmitry Bulgin 7 , Sergei Orlov ${ }^{7}$, Pavel Musienko ${ }^{4,5,6}$, Stanisa Raspopovic ${ }^{8 * *}$ and Marco Capogrosso $3,9,10,11 * *$

${ }^{1}$ School of Electrical Engineering, University of Belgrade, Serbia

2 The Mihajlo Pupin Institute, University of Belgrade, Serbia

${ }^{3}$ Rehab and Neural Engineering Labs, University of Pittsburgh, Pittsburgh, PA, USA

${ }^{4}$ Institute of Translational Biomedicine, Saint-Petersburg State University, Saint-Petersburg, Russia

${ }^{5}$ Pavlov Institute of Physiology, Russian Academy of Sciences, Saint Petersburg, 199034, Russia

${ }^{6}$ Sirius National Technical University, Neuroscience Program, Sochi, Russia

${ }^{7}$ Institute of Medical Primatology, Ministry of Science and Higher Education of the Russian Federation, Sochi, Russia

${ }^{8}$ Laboratory for Neuroengineering, Institute for Robotics and Intelligent Systems, ETH Zürich, Zürich, Switzerland

${ }^{9}$ Department of Neurological Surgery, University of Pittsburgh, Pittsburgh, PA, USA

${ }^{10}$ Department of Bioengineering, University of Pittsburgh, Pittsburgh, PA, USA

${ }^{11}$ Department of Physical Medicine and Rehabilitation, University of Pittsburgh, Pittsburgh, PA, USA

* Co-first authors

${ }^{* *}$ Co-senior authors

\begin{abstract}
Restoration of proprioception with neurotechnology is critical to improve effectiveness of robotic neuro-prostheses. Unfortunately, after initial enthusiasm clinical results showed that unlike touch, proprioception could not be reliably induced. Here we show that concurrent activation of multiple sensory modalities may trigger unwanted sensory regulation mechanisms that disrupt proprioception. We recorded intra-spinal neural activity induced by stimulation of proprioceptive afferents from the radial nerve in three monkeys. Then, we superimposed stimulation of the radial nerve cutaneous branch and quantified its impact on spinal neural activity via population analysis. Proprioceptive pulses produced robust neural trajectories in the neural manifold that were disrupted by concurrent stimulation of cutaneous afferents. This disruption correlated with a reduction of afferent volleys and multi-unit activity both in the spinal cord and somatosensory cortex. Our results suggest that limited specificity not only impacts localization of artificial percepts, but also their nature to an extent that was never considered.
\end{abstract}




\section{Introduction}

Somatosensory feedback is a critical requirement for motor control and coordination. Hence, neuroprosthetic systems aiming at restoring motor function with robotic devices have been designed to include some form of sensory feedback via neural interfaces ${ }^{1}$. Artificial somatosensory percepts that resemble touch have been successfully induced with electrical stimulation of the peripheral nerves ${ }^{2}$, spinal cord $^{3}$ and sensory cortex ${ }^{4,5}$. These percepts can be modulated with stimulation parameters in real time and have shown to improve effectiveness of prosthetic devices ${ }^{6-8}$ while decreasing mental and physical fatigue ${ }^{9}$. Indeed, since pioneering works $^{10}$ in animals, multiple independent clinical studies showed that electrical stimulation of peripheral sensory afferents through epineural ${ }^{7}$ and intraneural ${ }^{2,8,9,11,12}$ nerve stimulation, non-invasive transcutaneous stimulation ${ }^{13}$, and even dorsal root stimulation ${ }^{14}$ could elicit localized and graded somato-sensations on the missing limb of people with amputations. In contrast, reports on the ability to elicit graded and controllable proprioception have been remarkably rare (Table 1).

Table 1, Review of the reported sensations in examined studies with electrical stimulation. Peripheral nerve stimulation of upper-limb amputees using CUFF ${ }^{15,16}, \mathrm{FINE}^{7,17}$, TIME $^{18,19}$, Wire LIFE ${ }^{20-22}$, tf - LIFE ${ }^{16,23}$ and USEA ${ }^{8,22,24,25}$ electrodes. Peripheral nerve stimulation with lower-limb amputees using $\mathrm{FINE}^{26}$ and $\mathrm{TIME}^{9,11}$ electrodes

\begin{tabular}{l|c|c|c}
\hline & $\begin{array}{c}\text { Included } \\
\text { subjects = 35 }\end{array}$ & $\begin{array}{c}\text { Common= majority of responding } \\
\text { Rare= }<10 \% \text { of responding contacts }\end{array}$ & $\begin{array}{c}\text { Graded= Sensation } \\
\text { proportional to } \\
\text { charge/frequency }\end{array}$ \\
\hline Type of percepts & Reported in & Number of contacts & Properties \\
\hline Touch/pressure & $35 / 35$ & Common & Graded/Controllable \\
Tingling/paresthesia & $22 / 35$ & Common & Graded/Controllable \\
Proprioception & $\mathbf{2 7 / 3 5}$ & Rare & Episodic-not quantified \\
Temperature & $4 / 35$ & Rare & Episodic-not quantified \\
Pain & $3 / 35$ & Rare & Episodic-not quantified
\end{tabular}

Unfortunately, the lack of proprioception severely limits the usability of robotic limbs by preventing subjects from understanding the position of arm and fingers in space or ankle/knee angles during locomotion.

This overwhelming body of experimental evidence (Table 1) comes in stark contrast with intuitive understanding of electrophysiology. Indeed, large-diameter proprioceptive afferents should have the lowest threshold for electrical stimulation of the peripheral nerves. Therefore, they should be the easiest sensory afferents to recruit with neural interfaces ${ }^{27-31}$ before eliciting any cutaneous percept. Given the discrepancy between experimental findings and theoretical considerations, we hypothesized that may exist neurophysiological constraints that prevent the generation of functional proprioceptive percepts. In fact, every pulse of electrical stimulation recruits myelinated axons with an efficiency that is inversely proportional to the distance from the electrode and directly proportional to the fiber diameter ${ }^{30}$. In consequence, since fibers of different diameters are densely packed within nerve fascicles, practical implementation of electrical neurostimulation systems would always result, at least partially, in the recruitment of mixed diameter fiber distributions, hence, different sensory modalities ${ }^{28,32,33}$. Therefore, cutaneous receptors are recruited concurrently to larger diameter afferents such as golgi (Ib) and spindle (la) afferents ${ }^{34}$. These fibers converge to interneurons in the spinal cord where they undergo the first layer of sensory processing, and project to the gracilis (lower limb) or cuneate (upper limb) nuclei in the medulla oblungata, thalamus and then cortex ${ }^{35}$.

The last 90 years of research have shown that different sensory modalities interact with each other throughout this pathway with mechanisms such as pre-synaptic inhibition ${ }^{35-37}$ that regulate transmission of action potentials to post-synaptic targets. Therefore, we questioned whether non-specific recruitment of multiple-sensory modalities that is commonly produced 
with neuroproshtetic systems may trigger undesired phenomena of sensory-interference, which could hinder neural processing of proprioceptive percepts. To address this question, we designed a series of electrophysiology experiments in anaesthetized monkeys who share significant neuroanatomical and biomechanical similarity with the human upper limb. Specifically, we induced proprioceptive percepts from the hand and forearm by stimulation of the muscle branch of the radial nerve that does not contain cutaneous afferents ${ }^{37,38}$ using cuff electrodes. We recorded evoked proprioceptive neural signals both in the cervical spinal cord and somatosensory cortex. Then, we studied how concurrent stimulation of somato-sensory afferents in the cutaneous branch of the radial nerve impacted the spinal and cortical proprioceptive responses. Specifically, we examined dorso-ventral intra-spinal activity throughout a whole spinal segment using neural population analysis ${ }^{39}$. We detected multi-unit threshold crossing in response to proprioceptive pulses and performed dimensionality reduction to observe the spinal neural trajectories produced by proprioceptive percepts. Concurrent stimulation of the cutaneous afferents disrupted these neural trajectories suggesting a significant degradation of proprioceptive information in the spinal cord. These results were confirmed by classic electrophysiology analyses such as peak-to-peak amplitude of afferent volleys and multi-unit peri-stimulus time histograms in both the spinal cord and somatosensory cortex.

Our results suggest that lack of specificity in the recruitment of fiber modalities may reduce proprioceptive information by triggering unwanted suppression of the proprioceptive signals.

\section{Results}

\section{Simultaneous brain and spinal neural recordings of artificial sensory percepts}

To study the transmission of artificially induced proprioceptive percepts from the periphery to the cerebral cortex, we recorded intra-spinal neural signals (Figure 1) in the cervical spinal cord and intra-cortical neural signals from the somatosensory cortex of the forearm/hand (Area S1/S2, Extended Data Figure 1a). We extracted intra-spinal neural signals from a dorsoventral 32-channel linear probe that we implanted in the area just rostral to the innervation of the deep radial nerve (C5 Segment). Because proprioceptive signals enter the spinal cord from the dorsal aspect and project towards medial and ventral laminae ${ }^{35}$, probe direction was consistent with dorso-ventral segment geometry allowing us to extract neural information from a whole section of a spinal segment. This enabled us to perform neural population analysis of the dorso-ventral signal processing path. The radial nerve, carrying sensory signals from the dorsal part of the forearm and hand, splits in proximity of the elbow into a pure-muscle and a pure-cutaneous branch, e.g., the deep and superficial branches of the radial nerve ${ }^{37}$ offering the opportunity to provide with modality-selective sensory stimuli. We implanted cuff electrodes on these two branches to be able to generate either proprioceptive or cutaneous percepts via electrical stimulation. We tuned the stimulation amplitude of the cutaneous nerve by observing the emergence of clear sensory volleys in the dorsal spinal cord in response to low-frequency stimulation (Extended Data Figure 1b). Instead, since the muscle nerve is a mixed proprioceptive/motor nerve, we ensured that only largest diameter sensory afferents were recruited by tuning the stimulation amplitude to sub-motor threshold while obtaining robust volleys in the spinal cord (Extended Data Figure 1c). We generated proprioceptive percepts by stimulating the muscle nerve at low frequency $(\sim 2 \mathrm{~Hz})$, to examine at least $500 \mathrm{~ms}$ of neural signals post stimulation. Instead, we provided cutaneous stimulation as continuous $\sim 50 \mathrm{~Hz}$ pulses, which is a typical configuration in sensory neuroprostehetic applications.

In summary, we recorded neural signals in the spinal cord and the somatosensory cortex of three Macaca Fascicularis monkeys while stimulating only proprioceptive, or concurrently proprioceptive and cutaneous afferents. 


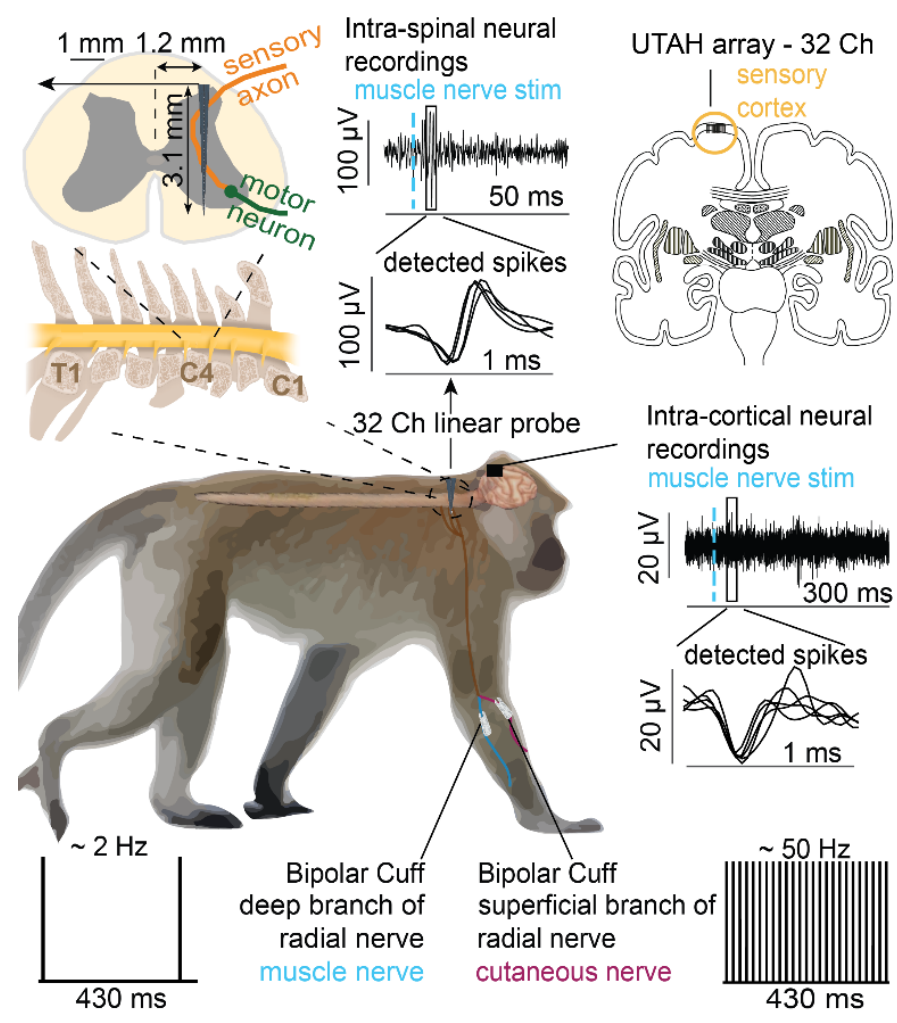

Figure 1, Experimental setup: Schematic illustration of experiments. Stimulation: we implanted two nerve cuffs for stimulation on the superficial branch (cutaneous nerve) and the deep branch (muscle, proprioceptive nerve) of the radial nerve. We stimulated muscle nerve at $\sim 2 \mathrm{~Hz}$, exclusively or concurrently with $\sim 50 \mathrm{~Hz}$ stimulation of the cutaneous nerve branch. Recording: we recorded neural activity with a 32-channel multi-electrode array in the somatosensory cortex and a 32-channel dorso-ventral linear probe implanted in the gray matter of the spinal segment C5. Top right and top left panels show typical neural responses induced by stimulation of the muscle nerve. Zoom insets show detected spikes waveforms, e.g., single neurons responses to proprioceptive inputs.

\section{Proprioceptive inputs elicit robust trajectories in the spinal neural manifold}

Sensory afferents enter the spinal cord from the dorsal horn and project to interneurons in the intermediate and ventral laminae. Therefore, the flow of information follows a dorso-ventral pathway. We utilized population analysis of our dorso-ventral linear probe to unveil the latent properties of the spinal neural processing. Indeed, population analysis allows to appreciate the dynamics of a neural system beyond single-unit properties through dimensionality reduction of the firing rates from the entire probe ${ }^{40}$, thus providing a mean to analyze and quantify the processing of proprioceptive activity within a whole spinal segment (Figure 2a).

We analyzed intra-spinal neural signals in response to stimulation of the muscle nerve at $2 \mathrm{~Hz}$ and extracted firing rates over a time window of $430 \mathrm{~ms}$ around each stimulation pulse (to avoid overlap with next pulse and including a pre-simuli window, Figure 2d). We utilized Principal Component Analysis of the multi-unit firing rates of the whole probe and identified "independent variables", or neural modes ${ }^{39,41}$ that explained most of the variance within a lowdimensional space ${ }^{40-42}$ (Figure 2a). In our analysis, 3 dimensions were sufficient to explain $65 \%$ of the variance in the signals, which is similar to that obtained in brain neural analysis $39,41,43$.

In the spinal manifold, the neural firing rates followed very precise behaviors after each stimulation pulse eliciting closed trajectories that were highly reproducible (Figure 2d). Given 
the robustness and reproducibility of these trajectories, we hypothesized that estimated trajectory lengths could be used as a proxy to measure the amount of proprioceptive information processed within the segment.

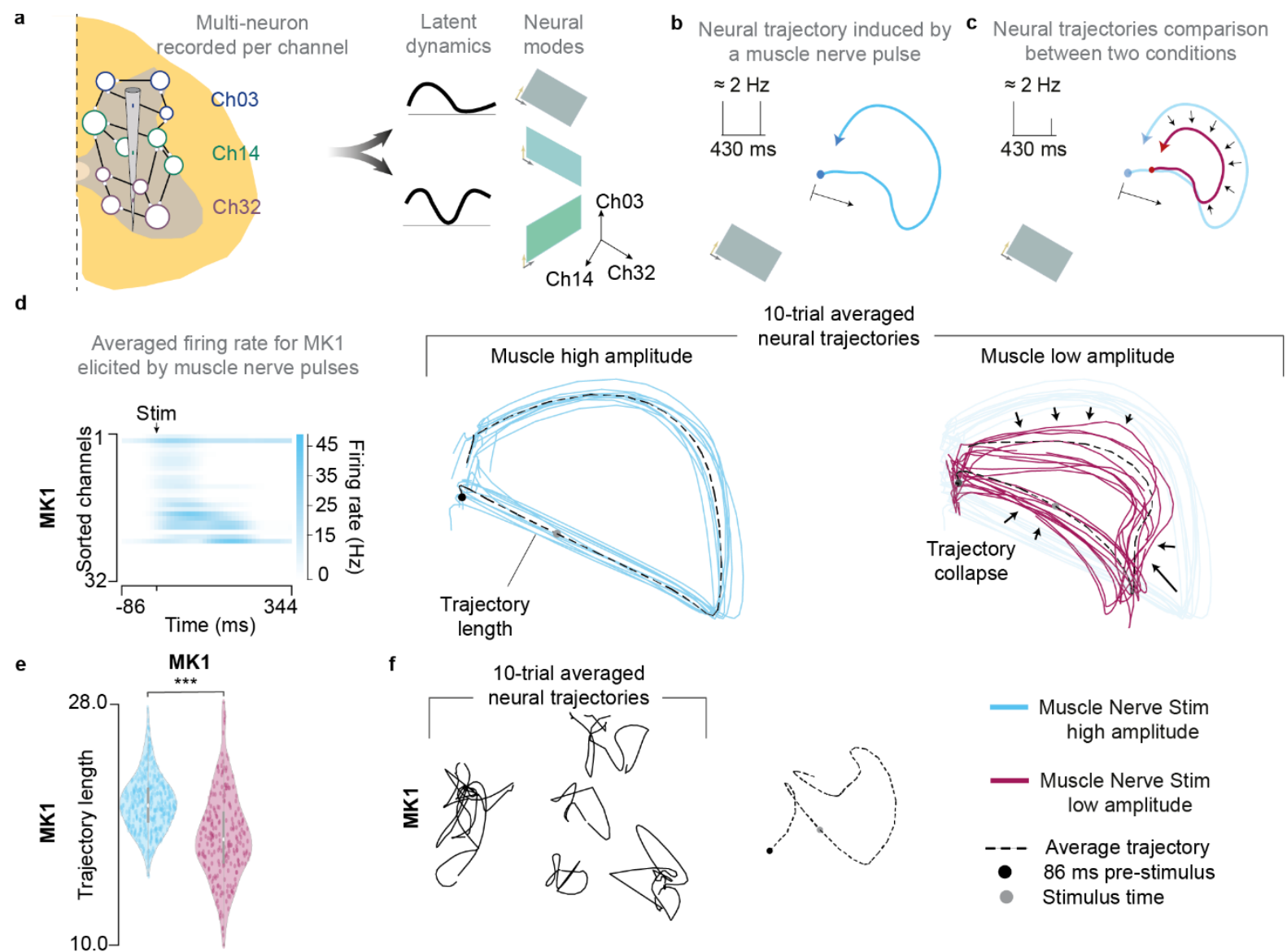

Figure 2, Intra-spinal neural population analysis. a) Latent dynamics and neural modes obtained from the multi-neuron recorded per channel. Principal components analysis of the neural activity recorded from the gray matter of the spinal cord identified the neural modes that defined the low-dimensional space from the 32-channel spinal probe. In these subspaces, neural activity followed precise latent dynamics. In a neural manifold, b) representation of a neural trajectory elicited by a muscle nerve pulse and c) the shrinking of this trajectory due to a low amplitude stimulation of the muscle afferents. d) Averaged multi-unit firing rate across all 32 channels for MK1 and its resultant 10-trial averaged neural trajectories elicited by muscle nerve stimulation both at a high and low stimulation amplitude to appreciate the phenomenon of trajectory collapse. e) Statistical quantification of the trajectory length for all monkeys for high and low stimulation amplitude of the muscle nerve ${ }^{* * *} \mathrm{P}<0.005$, Kruskal-Wallis test with 380 and 231 points for high and low amplitude, respectively, for MK1). Violin plots: each dot corresponds to the computed trajectory length for a trial, forming a Gaussian distribution of trajectory lengths. The central mark represented as a white dot indicates the median, and the gray line indicates the 25th and 75th percentiles. The whiskers extend to the most extreme data points not considered outliers. f) 10-trial averaged neural trajectories (left) and total averaged trajectory (right) for cutaneous nerve stimulation at high stimulation amplitude. Trial corresponds to a stimulation pulse.

Therefore, we projected these trajectories on hyperplanes called neural manifolds, which were defined by the neural modes, and calculated the length of these trajectories in the neural space for each monkey and each condition (Figure 2b). Since these trajectories were the result of the perturbation induced by proprioceptive inputs in the spinal neural networks, we loosely interpreted this variable as a measure of the amount of "energy" that each artificial proprioceptive percept elicited in the examined spinal circuits. Because the amount of "energy" 
should be proportional to the stimulation amplitude, which controls the number of recruited afferents, we tested the validity of this measure by computing the neural trajectories induced by proprioceptive inputs both at high and low amplitudes (Figure 2d). We found that muscle nerve stimulation at a high amplitude elicited longer trajectories, thereby conveying a higher amount of "energy" and vice versa. This observation was consistent in MK1 and MK2 (Figure 2e, Extended Data Figure 3) but not in MK3, probably as a consequence of the variability of neural data at low amplitude in this animal (Extended Data Figure 3).

To further control that these trajectories were a non-trivial feature of the stimulation, we also computed trajectories elicited by cutaneous nerve stimulation. In contrast to proprioceptive trajectories, cutaneous neural trajectories were extremely variable and did not reproduce closed trajectories (Figure 2f). This is consistent with the position of our probe that captures the dorso-ventral proprioceptive flow rather than the dorsal-horn specific processing of cutaneous inputs ${ }^{44}$.

In summary, we showed that population analysis of a dorso-ventral linear probe in the grey matter of the spinal cord shows highly robust and reproducible trajectories in the neural manifold in response to artificial proprioceptive pulses. We proposed to quantify the length of this trajectory as a mean to assess the amount of proprioceptive information flowing through the spinal cord and use this variable to evaluate the impact of artificial cutaneous input on proprioceptive information.

\section{Cutaneous nerve stimulation disrupts intra-spinal neural proprioceptive trajectories}

Sensory modalities interact with each other in the spinal cord and brainstem with gating mechanisms like pre-synaptic inhibition and primary afferent depolarization ${ }^{36}$, which regulate the flow of sensory and, particularly, proprioceptive inputs in the spinal cord. We hypothesized that artificial stimulation of cutaneous afferents may trigger these mechanisms and disturb the transmission of proprioceptive inputs. Since we previously showed that the length of the neural trajectories could be a proxy for the amount of "energy" induced by the proprioceptive inputs, we tested whether proprioceptive neural trajectories would shrink when concurrent stimulation of cutaneous afferents is super-imposed to proprioceptive inputs. Therefore, we projected proprioceptive trajectories on the neural manifolds elicited by stimulation of the proprioceptive branch (Figure 3a) and those elicited by stimulation of the proprioceptive branch while concurrently stimulating the cutaneous branch at $50 \mathrm{~Hz}$, a frequency commonly used in sensory neuroprosthetics.

All three monkeys exhibited robust trajectories in response to proprioceptive inputs and, in all three monkeys, concurrent stimulation of cutaneous afferents significantly reduced trajectory lengths (Figure 3b) albeit with different effect size. MK1 and MK3 exhibited the largest disruption, while MK2 was significantly disrupted but to a lesser extent. This result seems to indicate that concurrent stimulation of cutaneous afferents reduced the amount of proprioceptive processing in the spinal cord. To further validate this concept, we repeated the same experiment at a lower cutaneous stimulation amplitude, i.e., we recruited less cutaneous afferents. In this condition, according to our hypothesis, the interference should be less pronounced provided that less cutaneous fibers are artificially active. As expected, low 
amplitude cutaneous stimulation increased trajectory length, thus reducing cancellation significantly in all monkeys.

a
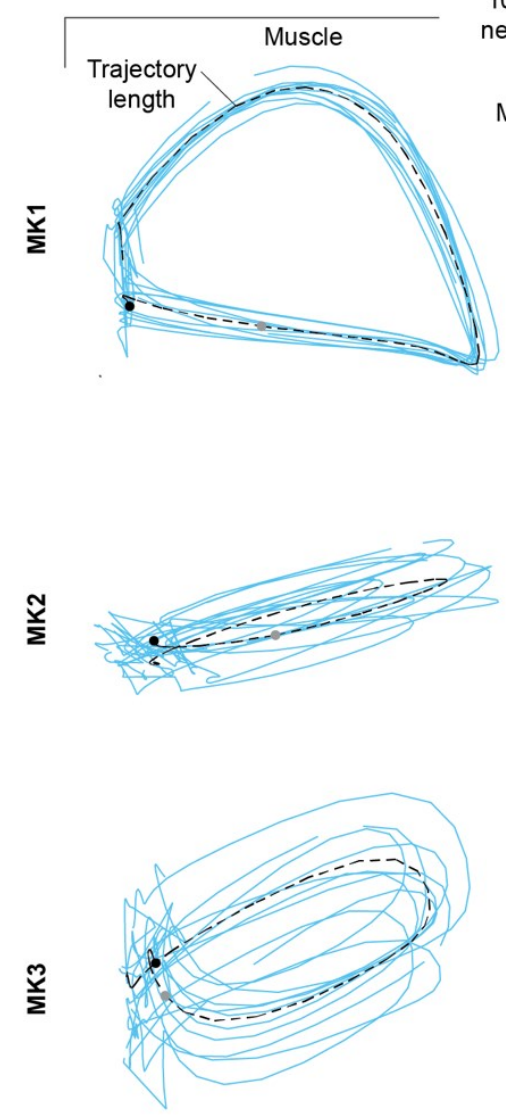

$\begin{array}{cc}-- & \text { Average trajectory } \\ - & 86 \text { ms pre-stimulus }\end{array}$

Stimulus time
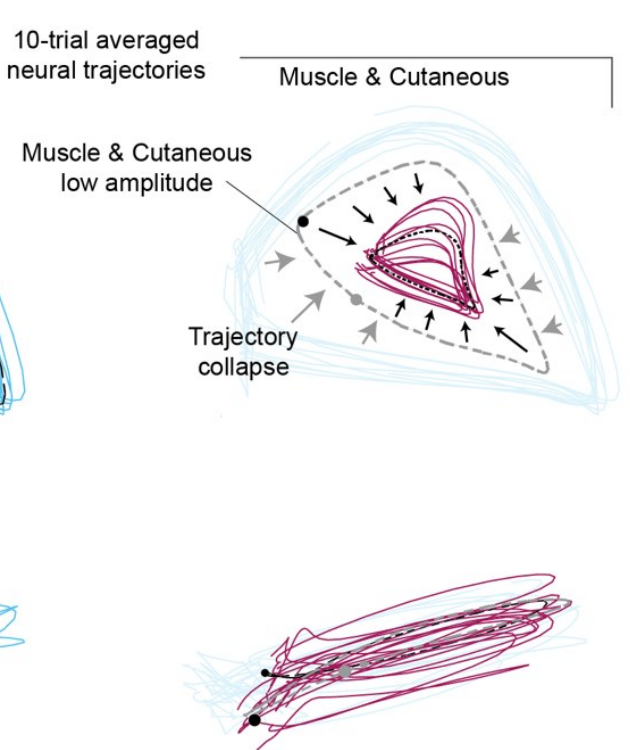

_ Muscle Nerve Stim

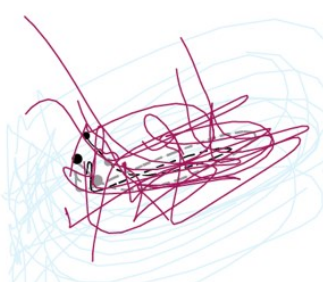

- Muscle \& Cutaneous Nerves Stim
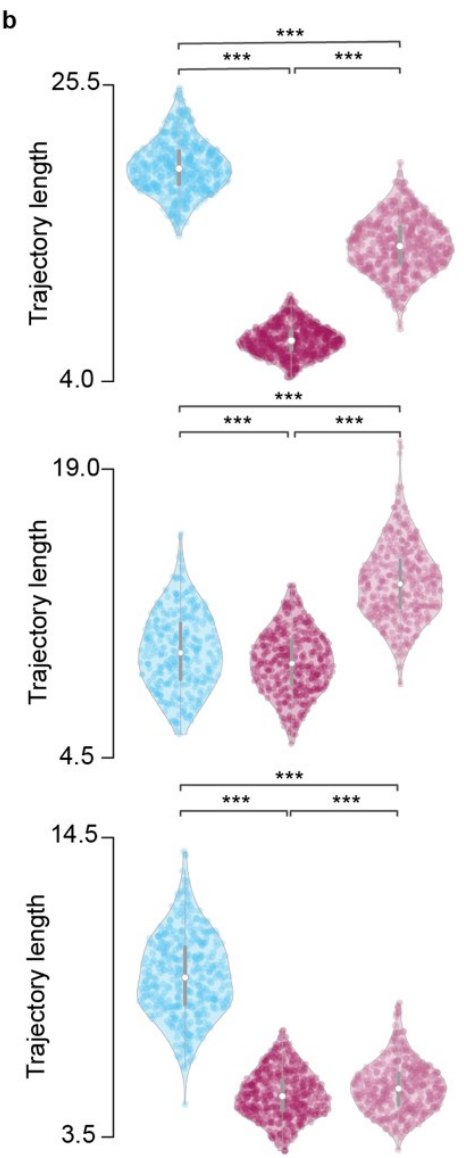
high amplitude

- Muscle \&Cutaneous Nerves Stim low amplitude

Figure 3, Neural trajectory lengths. a) Comparison of the neural trajectories induced by muscle nerve stimulation and concurrent cutaneous stimulation. Gray dashed lines indicate average trajectory for muscle and cutaneous nerves stimulation at a subthreshold amplitude. b) Statistical analysis of the trajectory lengths for each stimulation condition $\left({ }^{* * *} P<0.005\right.$, Kruskal-Wallis test with 381,470 and 453 points for muscle nerve stimulation, concurrent cutaneous stimulation at high amplitude and low amplitude, respectively, for MK1;369, 410 and 411 points, respectively, for MK2; 392, 380 and 371 points, respectively, for MK3). Violin plots: each dot corresponds to the computed trajectory length for a trial, forming a Gaussian distribution of trajectory lengths. The central mark represented as a white dot indicates the median, and the gray line indicates the 25th and 75th percentiles. The whiskers extend to the most extreme data points not considered outliers. Trial corresponds to a stimulation pulse.

These results, obtained with population analysis, were non-obvious if we consider that during proprioceptive and cutaneous stimulation the spinal cord was receiving significant more artificial input. Indeed, continuous stimulation of the cutaneous afferents significantly increased the total firing rates (Extended Data Figure 2), which is a trivial observation given that multiple cells fire in response to $50 \mathrm{~Hz}$ cutaneous volleys. However, the increase was not captured by the neural manifold because it was independent from proprioceptive input processing. Consistent with our interpretation, low amplitude cutaneous stimulation yielded lower firing rates than high-amplitude and yet exhibited longer proprioceptive neural trajectories. 
In summary, we showed that concurrent stimulation of the cutaneous nerve significantly suppressed proprioceptive neural trajectory lengths, suggesting that artificial recruitment of cutaneous afferents hinders processing of proprioceptive inputs in the spinal cord.

\section{Cutaneous nerve stimulation reduces proprioceptive afferent volleys}

The use of population analysis corroborated our hypothesis that concurrent stimulation of sensory modalities may cause a suppression of proprioceptive information processing in the spinal cord. We then sought to validate our results obtained by population analysis by verifying that observations performed on neural trajectories were paralleled by standard electrophysiological signatures of sensory input suppression. Therefore, we analyzed feature of the afferent volleys induced by proprioceptive pulses, and the firing rates of multi-units responding to proprioceptive pulses.
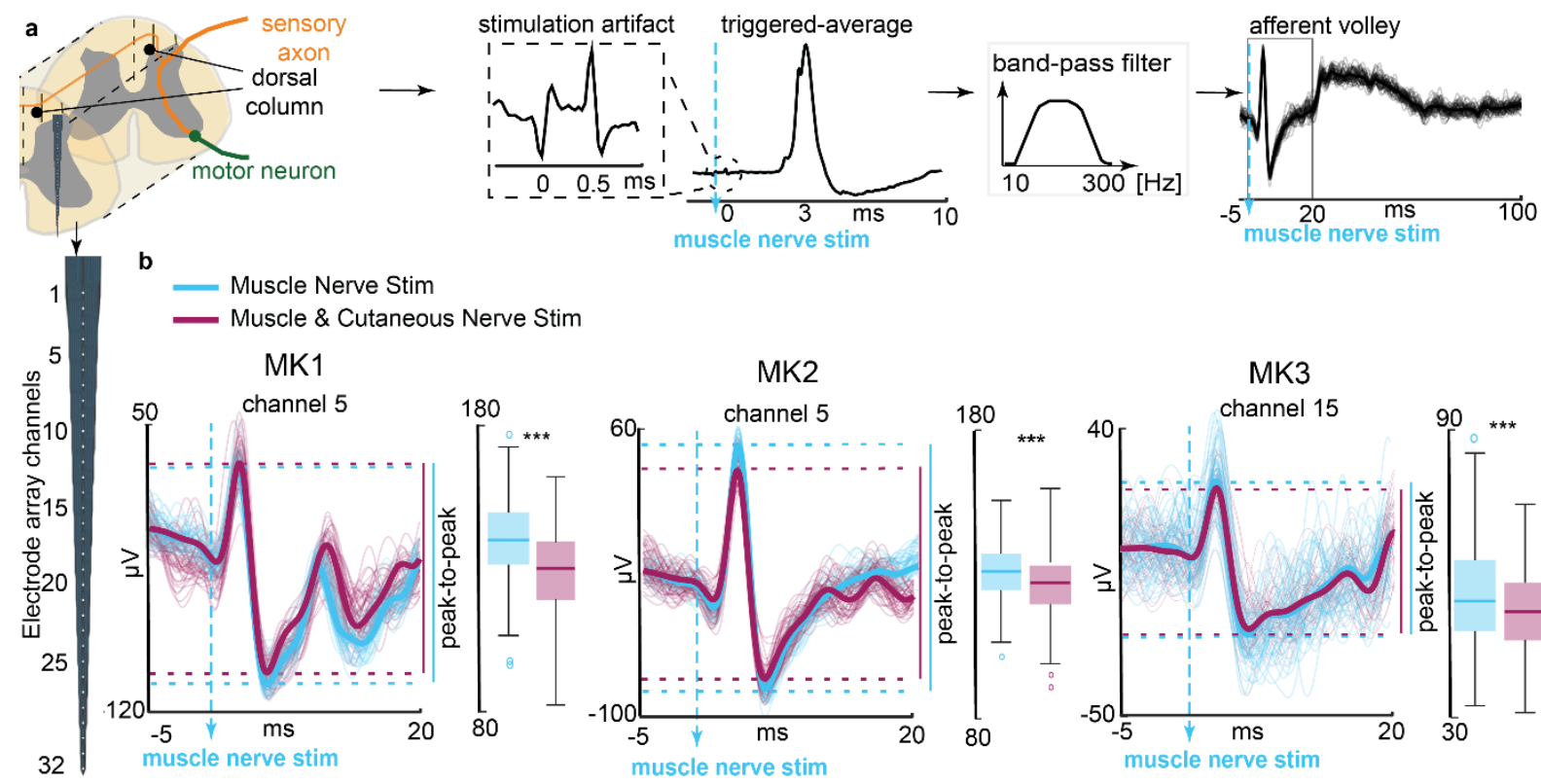

Figure 4, Proprioceptive afferent volley peak-to-peak amplitude suppression. a) Definition and extraction of the afferent volley. Triggered-average signal showed stimulation artifacts in the signal (zoomed insight) around the time of muscle nerve stimulation (pulse width: $0.5 \mathrm{~ms}$ ) while the afferent volley appeared $\approx 3.6 \mathrm{~ms}$ after the stimulation. We removed spiking component of the signal and analyzed the afferent volley during first $20 \mathrm{~ms}$ after the stimulation. b) Afferent volleys in three monkeys. Afferent volleys as a response to proprioceptive nerve stimulation, with concurrent cutaneous nerve stimulation (magenta) or without it (cyan). Transparent curves represent the individual response to each single stimulus repetition, while solid curves are averaged signals across all the individual responses. We compared peak-to-peak amplitude values of afferent volleys over 2 conditions with one-way ANOVA with 350 points, where each point represents the peak-to-peak amplitude as a response to a single stimulus pulse. Boxplots: The central mark indicates the median, and the bottom and top edges of the box indicate the 25th and 75th percentiles, respectively. The whiskers extend to the most extreme data points not considered outliers, and the outliers are plotted individually using the ' $o$ ' symbol. Asterisk: ${ }^{* * *} p<0.001$.

Signals from the periphery are projected through dorsal columns and spinocerebellar tracts to the medulla oblongata and other layers of the sensorimotor system. Several channels of implanted dorso-ventral intra-spinal linear probe were positioned in the dorsal horn, thus in proximity to the dorsal columns and capable to capture ascending afferent volleys elicited by stimulation of the proprioceptive afferents (Figure $\mathbf{4 a}$ ). If cutaneous stimulation would reduce 
proprioceptive input processing, then proprioceptive afferent volleys should show a reduction in peak-to-peak amplitude when cutaneous afferents are concurrently activated.

To test this hypothesis, we inspected stimulation triggered average signals defined as the mean neural response across single muscle nerve stimulation pulses. Simple signal analysis revealed strong, consistent volleys produced by a single-pulse stimulation of the proprioceptive branch of the radial nerve (Figure 4a) at a latency of about $3.6 \mathrm{~ms}$ after each proprioceptive pulse.

Cutaneous nerve stimulation reduced peak-to-peak amplitude of proprioceptive volleys in all three monkeys (Figure 4b). This suppression was visible in all the channels where we could record afferent volleys. In summary, we found that cutaneous nerve stimulation reduced peakto-peak proprioceptive afferent volleys, suggesting that proprioceptive information transmitted towards the upper layers of the sensorimotor system is reduced.

\section{Proprioceptive units show reduced firing rates during stimulation of the cutaneous afferents}

We then utilized multi-unit threshold crossing analysis (Figure 5a) and quantified the obtained neural activity in response to proprioceptive stimuli by analyzing the peristimulus time histogram (PSTH) using mean event rate as a measure to identify units that consistently responded to proprioceptive inputs. The PSTH represents the mean number of unit spikes that occurred within a defined time frame across all proprioceptive stimulation pulses (see Methods). In our data, peak of PSTH occurred at approximately $3-4 \mathrm{~ms}$ after each proprioceptive stimulation pulse which is expected considering conduction velocities in monkeys nerve afferents ${ }^{37}$.

Concurrent stimulation of cutaneous and muscle nerve suppressed the multiunit activity triggered with muscle stimulation. We found evidence of significantly reduced PSTH peak values in all three monkeys (Figure $5 \mathbf{b}$ ) and across multiple channels.

In summary, we found evidence in all animals that intra-spinal multi-unit activity in response to proprioceptive stimuli was significantly reduced during continuous stimulation of cutaneous afferents. 

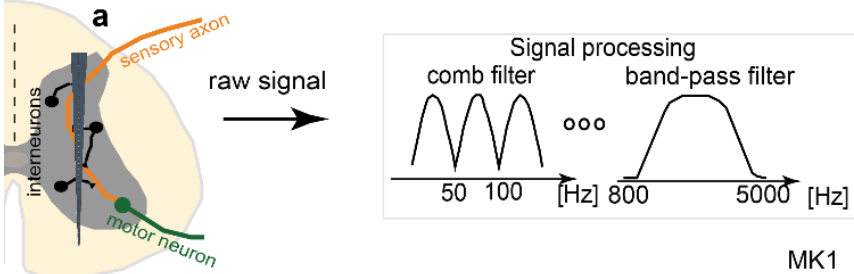

b

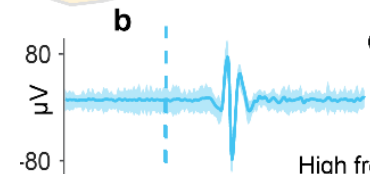

Channel 15

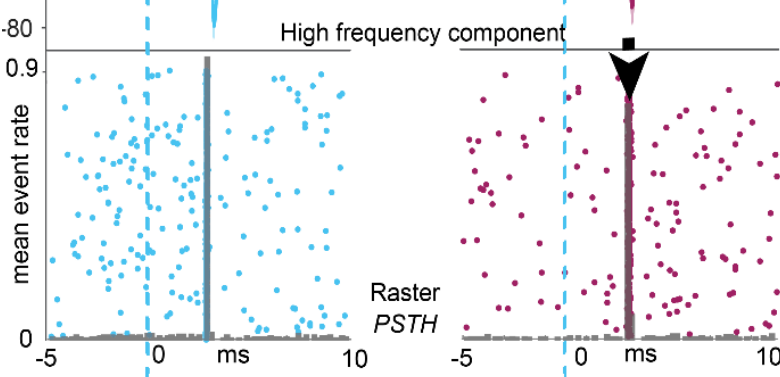

Channel 5
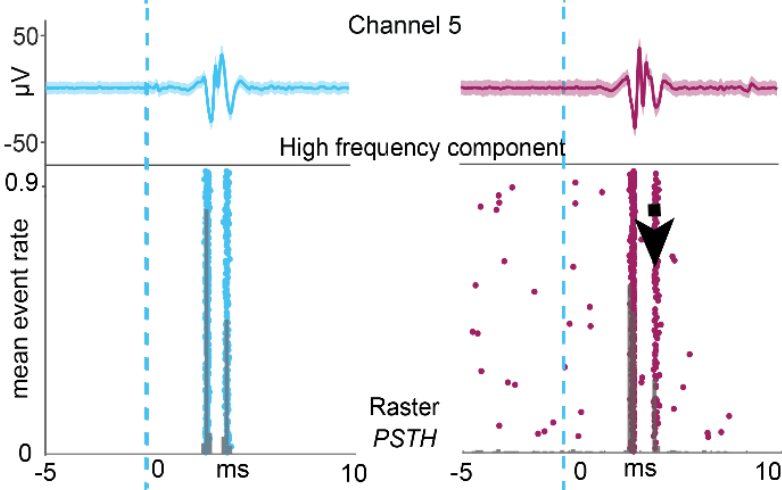

MK1

MK1

30

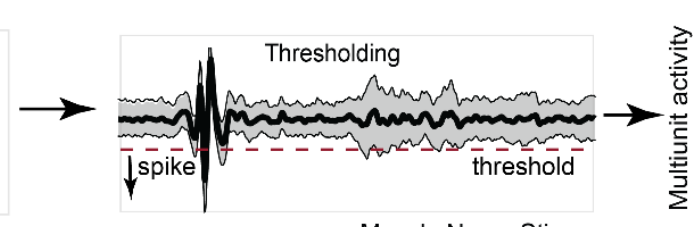

- Muscle Nerve Stim

- Muscle \& Cutaneous Nerve Stim

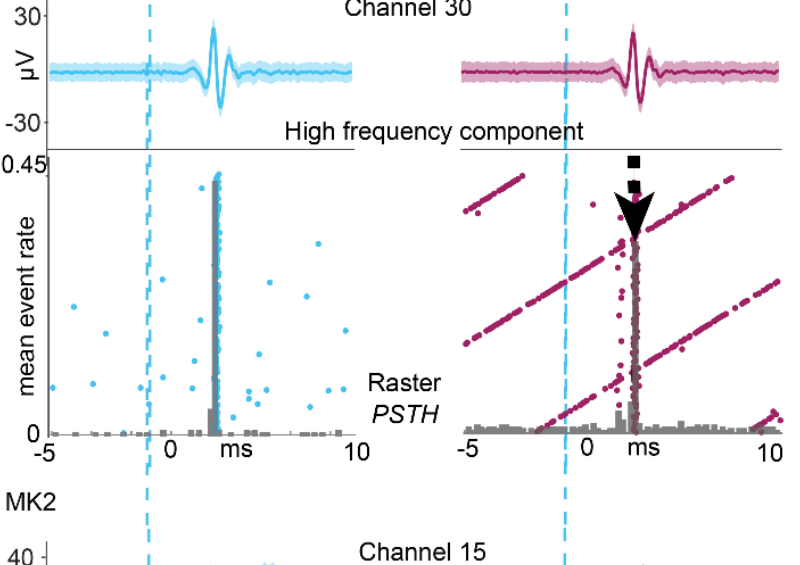
10
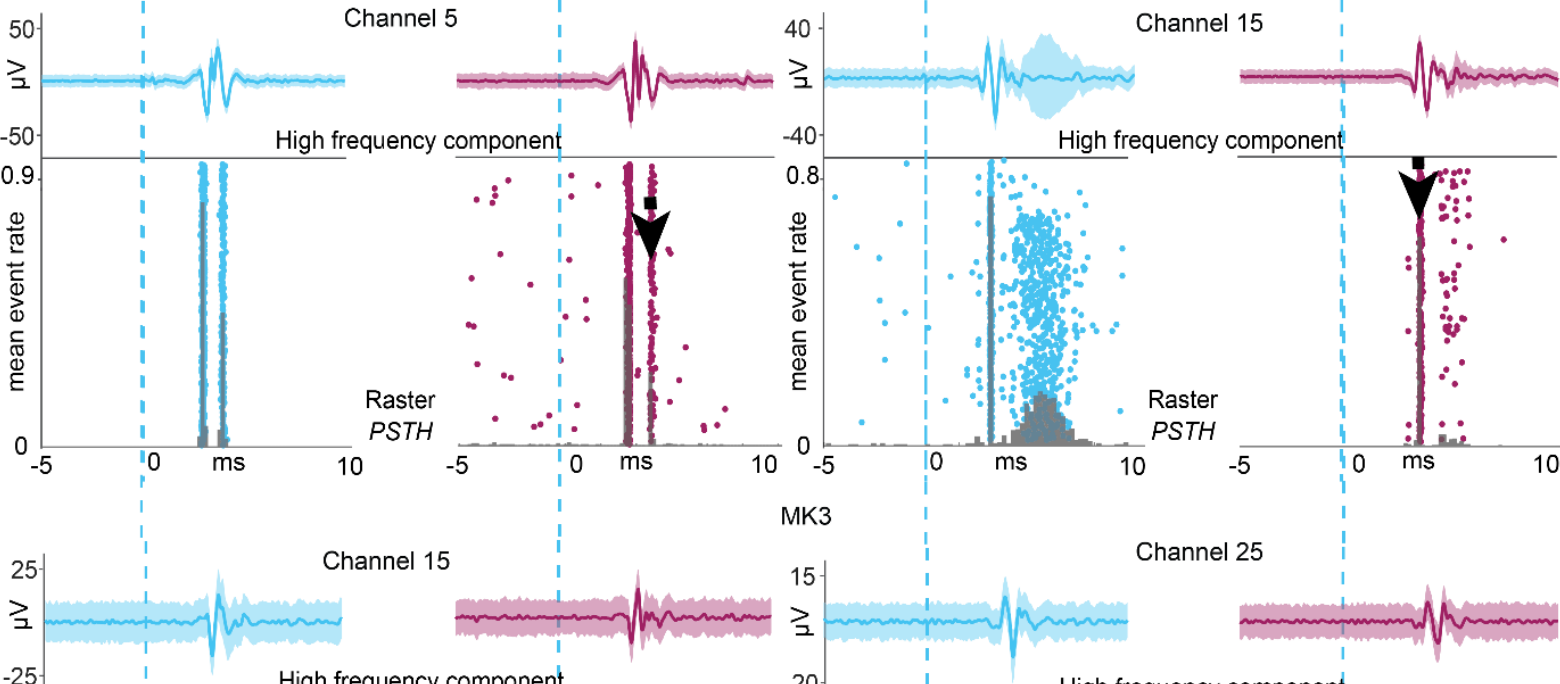

MK3
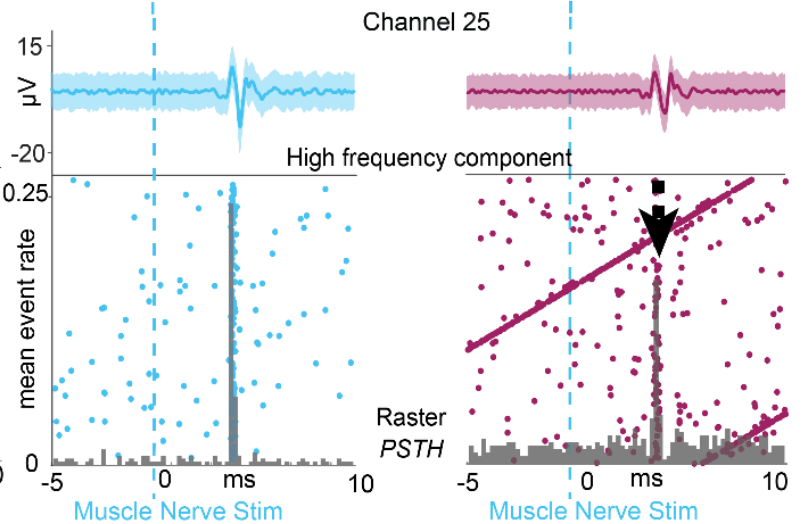

Figure 5, Multiunit activity suppression. a) Obtaining a multiunit neural activity. We filtered the signal to extract the spiking component and detect the neural action potentials using the thresholding algorithm. b) Multiunit activity and high-frequency components of two different channels for each of the three monkeys. Single muscle nerve stimulation (cyan, left) and simultaneous muscle and cutaneous nerve stimulation (magenta, right). Dashed cyan line represents the moment of muscle nerve stimulation pulse. Neural activity is presented and quantified with raster plot and peri-stimulus time histogram (PSTH). Each row of the raster plot represents the response to a single muscle nerve stimulation pulse, while each dot corresponds to an action potential. Mean event rate is defined as an average number of spikes within time frame of one bin $(0.2 \mathrm{~ms})$ across all single pulses of muscle nerve stimulation. In all presented examples, multiunit activity as a response to proprioceptive nerve stimulation is suppressed when cutaneous nerve is concurrently stimulated. 
bioRxiv preprint doi: https://doi.org/10.1101/2021.11.19.469209; this version posted November 20, 2021. The copyright holder for this preprint (which was not certified by peer review) is the author/funder, who has granted bioRxiv a license to display the preprint in perpetuity. It is made available under aCC-BY-NC-ND 4.0 International license.

\section{Reduction of proprioceptive information during stimulation of cutaneous afferents also occurs in cortex}

We showed that continuous stimulation of cutaneous afferents suppresses proprioception information processing in the spinal cord and correlates to classic electrophysiology assessments. While some of the sensory information bypasses spinal processing layers, presynaptic inhibition has been observed also in supra-spinal layers such as the brainstem ${ }^{36}$. Therefore, we hypothesized that intra-cortical neural responses to proprioceptive stimuli would also be reduced during concurrent stimulation of the cutaneous afferents, which would show a decrease in conscious perception of proprioception.

To test this hypothesis, we replicated our analysis on intra-cortical signals extracted from area 2 of the somatosensory cortex in MK1 and MK2 (Figure 6a) (MK3 cortical implant failed preventing this analysis). We found clear proprioceptive afferent volleys in cortex with a latency around $50 \mathrm{~ms}$, which is consistent with the further distance of the cortex from the peripheral nerves. Peak-to-peak analysis of the volley amplitude indicated statistically significant reduction of proprioceptive afferent volleys during concurrent suprathreshold stimulation of cutaneous nerve in both monkeys (Figure 6a). This suppression was observed in all the channels of the array (Figure 6a). Surprisingly, when we inspected the firing rates extracted from multi-units in the cortex, the rates obtained during low amplitude stimulation of the cutaneous nerve (less fibers) were higher in one monkey and similar in the other monkey to the rates obtained during high-amplitude stimulation of the cutaneous nerve. This is markedly different from what we observed in the spinal cord and counterintuitive. Indeed, we would have rather expected higher firing rates always associated to higher amplitude stimulation and not the opposite (Figure 6b, Extended Data Figure 4a, b). However, multi-unit PSTH analysis showed reduced intra-cortical multiunit activity of units responding to proprioceptive inputs during concurrent cutaneous stimulation, which paralleled the results obtained in the spinal cord.

In summary, we observed a reduction of proprioceptive information during continuous stimulation of the cutaneous nerve also in the somatosensory cortex. This finding suggests that the effects that we observed in the spinal cord propagate through the higher layers of sensorimotor processing. 


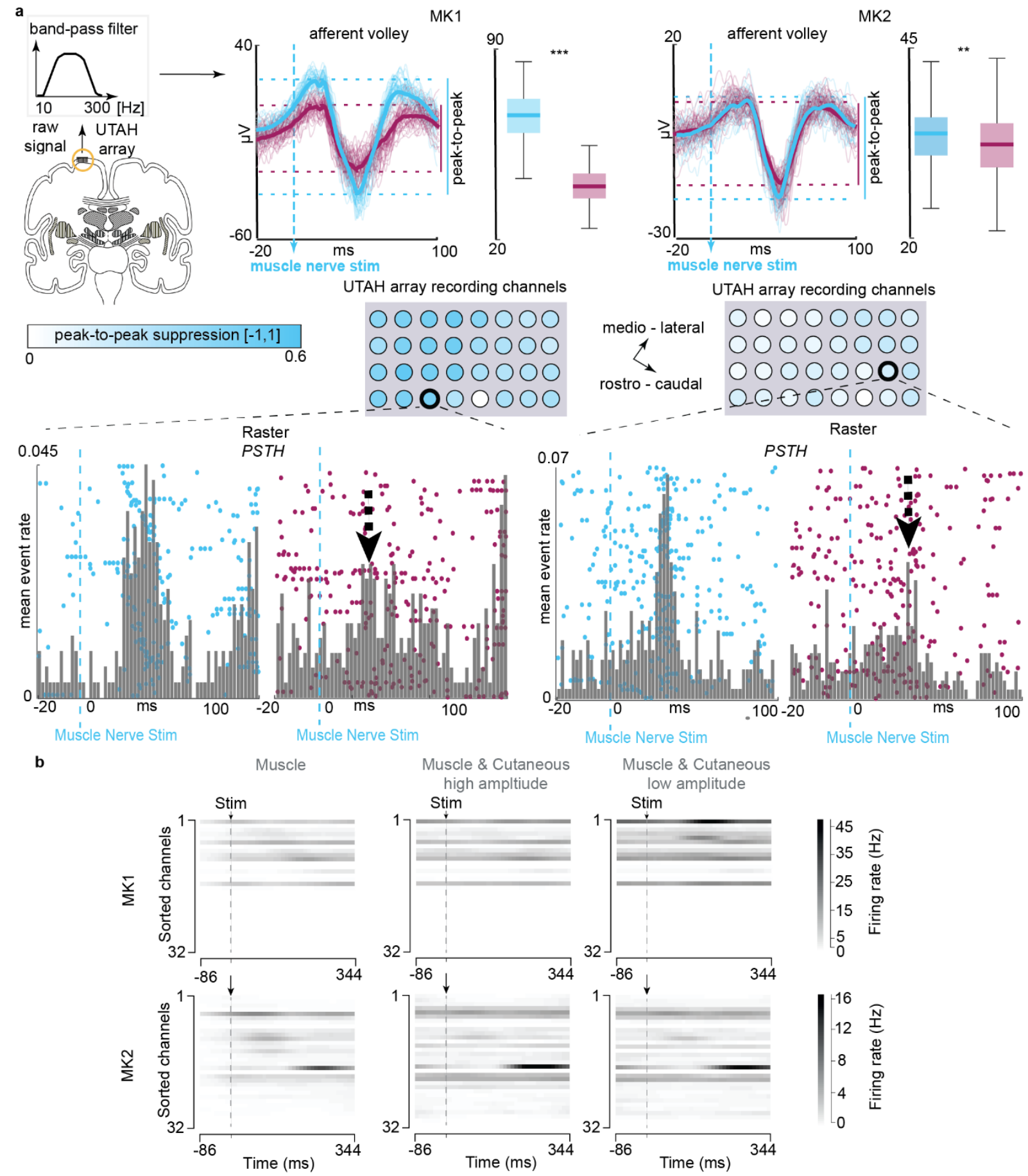

Figure 6, Cortical analysis. a) Proprioceptive afferent volleys peak-to-peak amplitude suppression in two monkeys. Transparent curves represent the individual response to each single stimulus repetition, solid curves are averaged volleys across all individual responses, with concurrent cutaneous nerve stimulation (magenta) or without it (cyan). Volleys are compared over 2 conditions with one-way ANOVA with 175 points for M1 and 180 points for M2 (point: peak-to-peak amplitude as a response to a single stimulus pulse). Boxplots: The central mark indicates the median, the bottom and top edges of the box indicate the 25th and 75th percentiles, respectively. The whiskers extend to the most extreme data points not considered outliers (asterisk: ${ }^{* * *} p<0.001,{ }^{* *} p<0.005$ ). Normalized peak-to-peak amplitude suppression presented for all UTAH electrode channels. Suppression is quantified as the ratio of peak-to-peak amplitude suppression of proprioceptive afferent volleys caused by simultaneous stimulation of the cutaneous nerve and peak-to-peak amplitude of single muscle nerve stimulation. Two examples of suppressed multiunit activity are given, presented and quantified with raster plot and peri-stimulus time histogram (1.6 ms bin). b) Averaged firing rate for each channel. Firing rate was averaged across all trials for each stimulation condition for MK1 and MK2. Channels were sorted according to the highest firing rate after the muscle stimulation pulse when the muscle nerve was stimulated. 


\section{Discussion}

In this study, we showed that neural processing of proprioceptive inputs was disrupted during concurrent continuous stimulation of cutaneous afferents. We observed evidence of this interference of sensory modalities in the spinal cord as well as upstream in the sensory cortex. Disruption of proprioceptive input may be attributable to the unwanted triggering of presynaptic inhibition of primary afferents or by the inability of common neural resources to process two distinct artificial information streams when simultaneously activated. Our results indicate that neurophysiological constraints within the network that are targeted by artificially recruited afferents may limit the efficacy of neuroprosthetic systems that aim to restore sensory feedback.

\section{Analysis of neural manifolds reveals disruption of intra-spinal processing of proprioceptive information}

We established a measure of proprioceptive neural information processing by quantifying proprioceptive neural trajectories in spinal neural manifolds using intra-spinal population analysis. Neural population analysis via neural manifold has been largely employed to study cortical processes $39,41,43$. Here we sought to use this method to quantify the impact of continuous stimulation of cutaneous afferents on spinal circuits while attempting to process proprioceptive information. In the spinal manifold, firing rates projected robust and closed trajectories in response to proprioceptive stimuli. Obviously, the robustness of our trajectories across animals stems from the fact that we produced artificial proprioceptive stimuli using electrical stimulation of the peripheral nerves, which produces strong synchronized volleys of activity rather than asynchronous burst of inputs ${ }^{45,46}$. Nevertheless, their reproducibility allowed us to appreciate quantitative changes in shape and features occurring at various conditions, i.e., reduced proprioceptive input or concurrent stimulation of cutaneous afferents. Since trajectory length was reduced when less proprioceptive afferents were recruited (low stimulation amplitude), we interpreted this feature as a proxy of the "energy" released by a proprioceptive input within the spinal circuits. As an analogy, we could think of trajectories in phase-space of physical oscillating systems such as pendulums that preserve their shape but change in size according to the energy in the system. Through the quantification of trajectory length, we assessed the effect of concurrent cutaneous stimulation on the spinal proprioceptive information and showed the collapse of proprioceptive neural trajectories during concurrent stimulation of cutaneous afferents, in other words, a suppression of proprioceptive information processed in the spinal cord. Importantly, simple analysis of total firing rates showed that the results of our manifold quantification were not trivial. Indeed, averaged multi-unit firing rates in response to proprioceptive stimuli were expectedly the highest during concurrent stimulation of the cutaneous afferents. This is an obvious result as general spinal activity is increased by the $50 \mathrm{~Hz}$ artificial cutaneous inputs. Thus, the actual total "energy" or activity in the spinal cord is higher during cutaneous stimulation. Yet the population analysis allows to extract only activity that explains the variance generated by proprioceptive inputs enabling to infer the proprioceptive components of the neural dynamics against the background of cutaneous activity. Hence, the use of neural manifolds for population activity analysis was crucial to assess phenomena of sensory interference.

We validated results obtained with neural manifold analysis with classical electrophysiology inspecting peak-to-peak afferent volleys as well as peri-stimulus time histogram analysis of proprioceptive units in the spinal cord. Both these measures indicated a reduction in proprioceptive information, thus validating our neural population analysis.

\section{Mechanisms underlying the suppression of proprioceptive inputs}

Pre-synaptic inhibition is one of the potential mechanisms that could explain our findings. Presynaptic inhibition is a well-known mechanism of sensory input gating that prevents transmission of excitatory post-synaptic potentials to neurons targeted by primary afferents ${ }^{36-}$ 
38. In our experiments, the stimulation amplitude of the proprioceptive nerve was the same across all conditions (e.g., fixed number of recruited afferents), therefore, the reduction of unit responses to proprioceptive inputs during cutaneous afferent stimulation is consistent with a reduction in synaptic inputs to these target units. In addition to pre-synaptic inhibition, there may be other mechanisms that could explain our data, for example, the "busy line" effect. Continuous, non-natural stimulation of the cutaneous afferents may produce highly synchronized activity in spinal interneurons (see for example Figure $\mathbf{5}$ MK1 channel 30 and MK3 channel 25 background activity). Some of these interneurons may receive both proprioceptive and cutaneous inputs. However, when artificially synchronized cutaneous inputs reach the spinal cord, they may saturate mixed interneurons' membrane and reduce their capacity to respond to additional inputs. When these neurons cannot be employed to process proprioceptive information, the neural network achieves a saturated state where no further processing can be carried out. This may explain why neural trajectory lengths for low amplitude cutaneous stimulation were longer, despite overall firing rates were lower.

We performed large part of our analysis in the spinal cord, which is the first important layer of sensory processing, particularly, in regards to proprioception. However, conscious perception is processed at various layer above the spinal cord and particularly in the brainstem ${ }^{44,47}$. In fact, pre-synaptic inhibition has been observed also in the brainstem ${ }^{36}$ suggesting that what we observed in the spinal cord may occur in any other neural structure that is directly targeted by recruited sensory afferents. Indeed, peak-to-peak afferent volleys as well as unit responses to proprioceptive stimuli were suppressed also in the sensory cortex area 2, which is known to integrate cutaneous and proprioceptive inputs ${ }^{48}$. Moreover, if cortical signals were independent from spinal and brainstem processes, when looking at the global cortical firing rates we would have expected higher firing rates during high-amplitude stimulation of the cutaneous nerve and lower firing rates during low amplitude stimulation of the cutaneous nerve. Instead, we found higher or similar firing rates when we used low-amplitude stimulation of the cutaneous nerve. This may be indicative of the fact that high amplitude stimulation may convey more cutaneous input but less proprioceptive input to the cortex because of sub-cortical cancellation. In contrast, lower cutaneous stimulation may mean less cutaneous input but more proprioceptive input to the cortex as a consequence of less cancellation occurring in sub-cortical structures.

These overall results support the conclusion that conscious perception of proprioception may be altered as well by sub-cortical interference. While this hypothesis cannot be tested in subjects with amputation because of their limb loss, recent data in humans with sensory incomplete spinal cord injury shows that epidural stimulation of the spinal cord, that also recruits sensory afferents ${ }^{14}$, reduces proprioception acuity during supra-threshold stimulation ${ }^{3}$. This result in humans further support our hypothesis and we believe that it demands further investigation.

\section{The importance of fiber selectivity in sensory neuroprosthetics}

Research in sensory neuroprosthetics has been largely focused on obtaining focused, localized and reproducible percepts. Because the peripheral nerve offers a relatively simple anatomical target with longitudinal fibers arranged in clusters (fascicles), different groups aimed at stimulating restricted spatial regions with the assumption that this would have led to more spatially selective percepts. While this approach has worked well for artificial cutaneous sensations it has largely failed for proprioception. We argue that our data shows that fiberclass selectivity is more important than what was intuitively thought because the failure to stimulate specific classes of afferents may lead to the triggering of natural sensory gating mechanisms that generates sensory interference. In fact, one could say that the problem of selectivity has been underestimated, and that lack of selectivity not only limits the localization and extent of percept, but it affects their nature even by disrupting some of the information. In a way this reasoning is consistent with the interpretations arguing for "bio-mimetic" stimulation ${ }^{49}$, but offering a new insight. Not only a truly "bio-mimetic" stimulation protocol would produce more natural sensations, but it may avoid the interference phenomena we 
observed, thus enabling perception. In support to our conjectures, human data show that the only device that reliably reported controllable proprioceptive percept is the penetrating, slanted UTAH array ${ }^{25,50}$. Indeed, the ability to recruit only few afferents near the electrode tips may be key to avoid sensory interference. Future devices and stimulation strategies should take sensory interference into account both in the design of electrode as well as in the design of stimulation protocols to achieve robust and reliable proprioception.

\section{Acknowledgements}

The authors would like to thank Sara Conti for her help during pilot experimental procedures. The authors would also like to thank all the staff at the Sochi Institute of Primatology for their relentless and competent work and for the many nights spent exchanging life stories and singing songs from all over the world on the black sea. The authors wish to thank Brian Dekleva for his useful inputs on the crafting on this manuscript.

\section{Funding}

The study was funded through the start-up funds from the department of Neurosurgery of the University of Pittsburgh to MC, through the Swiss National Science Foundation (SNSF) grant MOVEIT (no. 205321_197271) and Innosuisse grant (no. 47462.1 IP-ICT) to SR.

\section{Author Contributions}

M.C. and S.R. designed the study. P.M., N.P., D.B., S.O., D.S., M.C. and S.R. designed and performed the surgical procedures. P.M., M.C., S.R. and O.G. performed all the experiments. N.K. and J.M.B. analyzed the data and made the figures. M.C., S.R., PM and D.S, secured funding M.C. and S.R. supervised the study. N.K., J.M.B., S.R. and M.C. wrote the manuscript and all the authors contributed to its editing.

\section{Competing Interests}

MC and SR hold patents in relation to peripheral nerve stimulation. SR is the founder of SensArs, a company developing neural interfaces for the peripheral nervous system.

\section{Data availability}

All data will be available upon reasonable request to the corresponding author.

\section{Code availability}

All codes will be available upon reasonable request to the corresponding author. 


\section{Extended data}

a

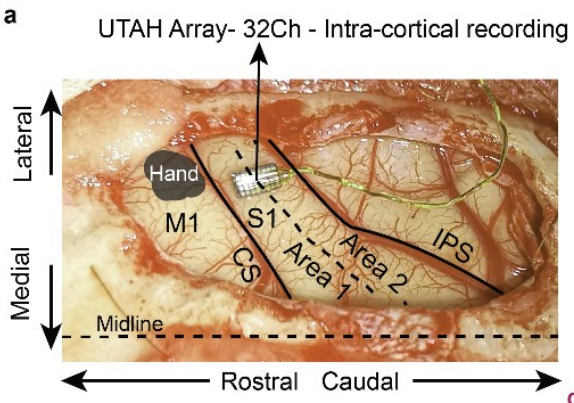

b

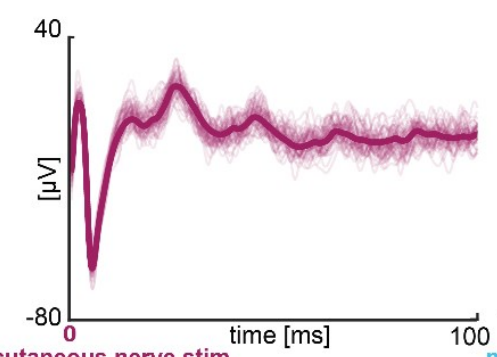

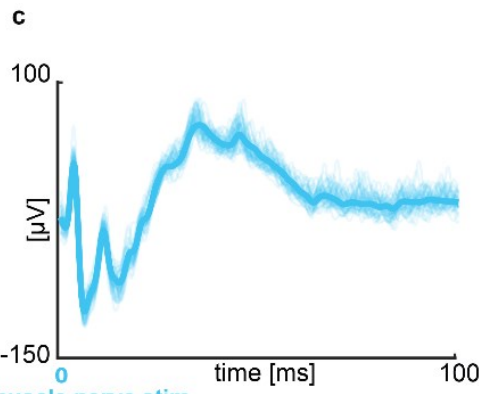

Extended Data Figure 1, Experimental procedure and electrophysiology details. a) representative picture showing the position of the UTAH array in relation to brain areas. We identified specific brain areas through anatomical landmarks and micro-stimulation of the cortex. We verified that a single pulse of stimulation delivered induced clear responses in the hand muscles. We determined the somatosensory area S1 in relation to the identified M1 anatomically and implanted the UTAH array electrode (Blackrock Microsystems) across Areas 1 and 2, b) Spinal cord recorded afferent volleys as a response to low-frequency cutaneous nerve stimulation. Stimulation amplitude of the superficial branch of the radial nerve is tuned as the one that is producing sensory volleys in the dorsal contacts of the linear spinal probes in response to low-frequency stimulation. c) Afferent volley recorded in spinal cord as a response to low-frequency muscle nerve stimulation. We tune threshold for stimulating deep branch of radial nerve to be able to evoke strong afferent volleys (right) while producing no or negligibly small M-waves in EMG recordings, in order to minimize direct activation of motor axons. 
a

b

c
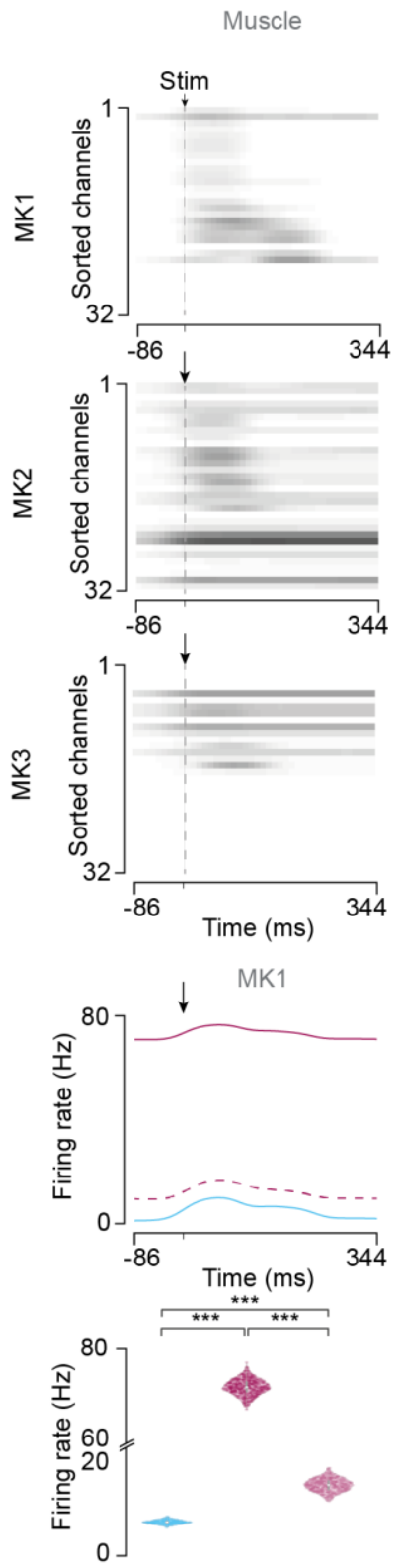

_ Muscle Nerve Stim
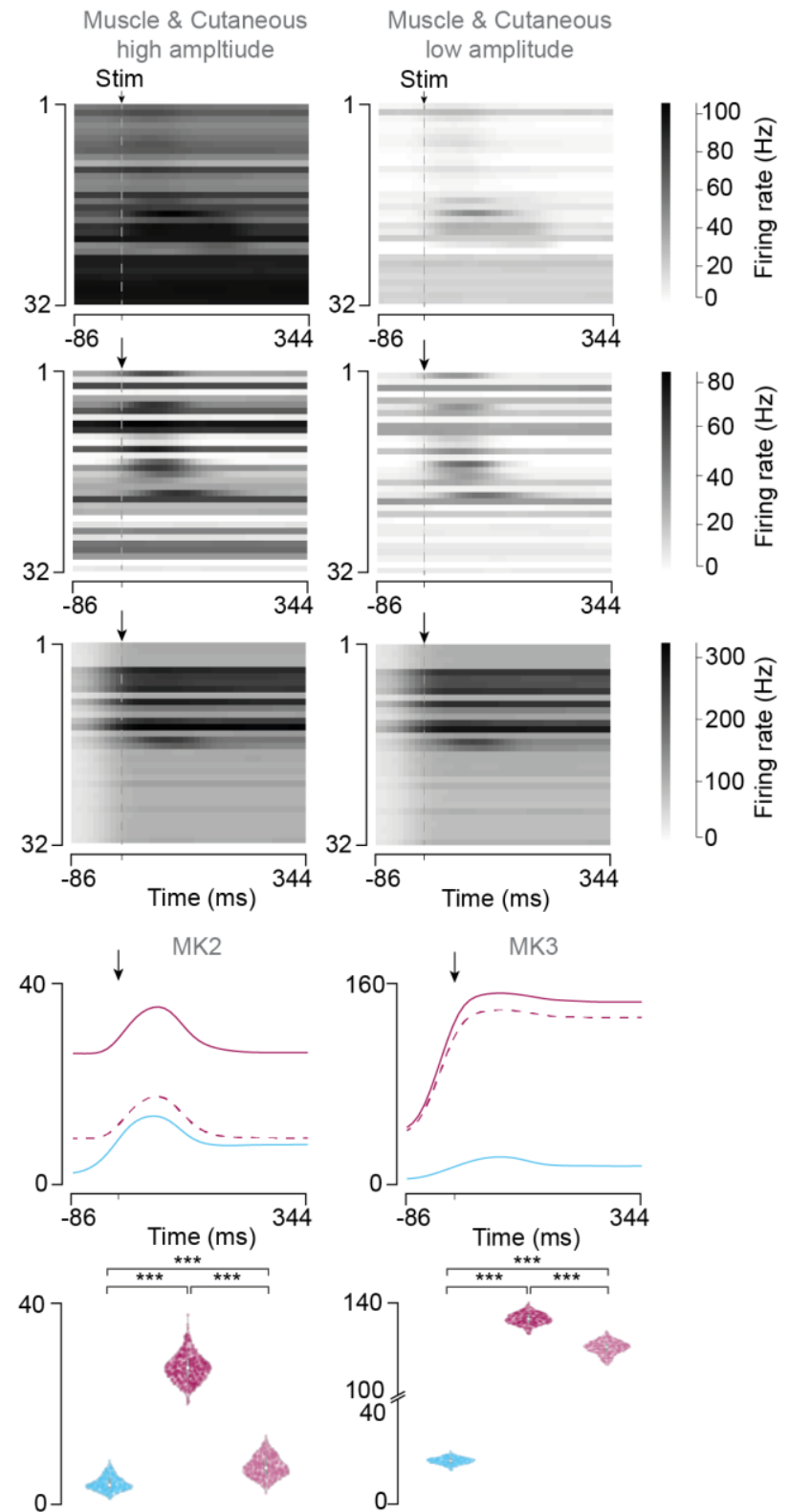

Muscle \& Cutaneous Nerves Stim high amplitude
Muscle \&Cutaneous Nerves Stim low amplitude

Extended Data Figure 2, Spinal firing rate. a) Averaged firing rate for each channel. Firing rate was averaged across all trials for each stimulation condition for three monkeys. Channels were sorted according to the highest firing rate after the muscle stimulus pulse when the muscle nerve was stimulated. b) Averaged firing rate. Firing rate was averaged across all trials and all channels for each stimulation condition for three monkeys. c) Statistical analysis of the firing rate for each stimulation condition ${ }^{* * *} \mathrm{P}<0.005$, Kruskal-Wallis test with 387,477 and 461 points for muscle nerve stimulation, concurrent cutaneous stimulation at high amplitude and low amplitude, respectively, for MK1; 374, 410 and 412 points, respectively, for MK2; 401, 388 and 376 points, respectively, for MK3). Violin plots: each dot corresponds to the computed trajectory length for a trial, forming a Gaussian distribution of trajectory lengths. The central mark represented as a white dot indicates the median, and the gray line indicates the 25th and 75th percentiles. The whiskers extend to the most extreme data points not considered outliers. Trial corresponds to a stimulation pulse. 


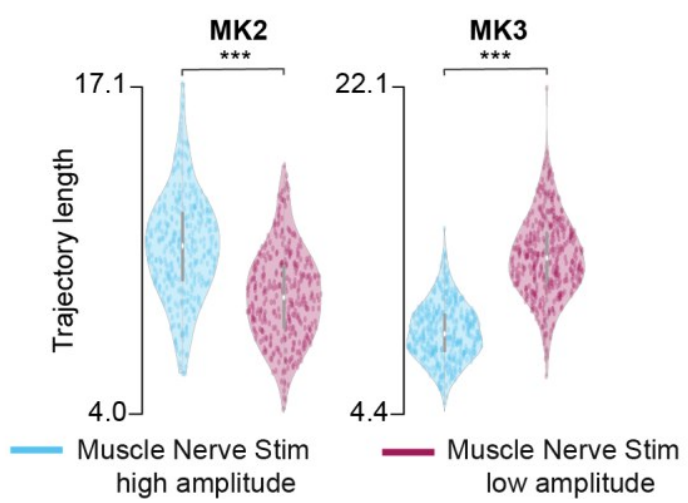

Extended Data Figure 3, Statistical analysis of the muscle nerve trajectory length. Statistical quantification of the trajectory length for all monkeys for high and low stimulation amplitude of the muscle nerve $\left({ }^{* * *} \mathrm{P}<0.005\right.$, Kruskal-Wallis test with 353 and 351 points for high and low amplitude, respectively, for MK2; 391 and 394 points, respectively, for MK3). Violin plots: each dot corresponds to the computed trajectory length for a trial, forming a Gaussian distribution of trajectory lengths. The central mark represented as a white dot indicates the median, and the gray line indicates the 25th and 75th percentiles. The whiskers extend to the most extreme data points not considered outliers. Trial corresponds to a stimulation pulse.

a

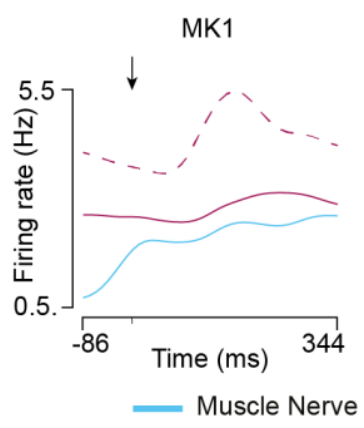

b

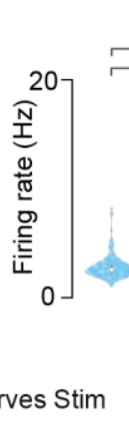

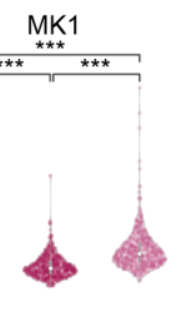

- - - Muscle \&Cutaneous Nerves Stim low amplitude

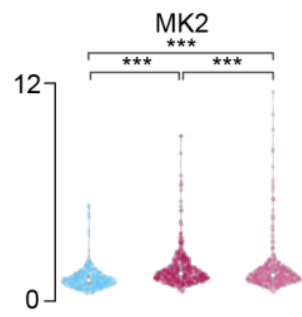

Extended Data Figure 4, Cortical firing rate. a) Total averaged firing rate. Firing rate was averaged across all trials and all channels for each stimulation condition for MK1 and MK2. b) Statistical analysis of the firing rate for each stimulation condition $\left({ }^{* *} \mathrm{P}<0.005\right.$, KruskalWallis test with 392, 474 and 460 points for muscle nerve stimulation, concurrent cutaneous stimulation at high amplitude and low amplitude, respectively, for MK2; 360, 389 and 392 points, respectively, for MK2). Violin plots: each dot corresponds to the computed trajectory length for a trial, forming a Gaussian distribution of trajectory lengths. The central mark represented as a white dot indicates the median, and the gray line indicates the 25th and 75th percentiles. The whiskers extend to the most extreme data points not considered outliers. Trial corresponds to a stimulation pulse. 


\section{Materials and methods}

\section{Animals}

The study was conducted according to the guidelines of the Declaration of Helsinki, and approved by the local Institutional Ethics Committee (protocol № 38/1, October 31, 2019).

Three adult Macaca Fascicularis monkeys were involved in the study (MK1 - MK 42286, male, 4 years old, $3.5 \mathrm{~kg}$, MK2 - MK 42588, male, 4 years old, $3.35 \mathrm{~kg}$, MK3 - MK 42328, male, 4 years old, $3.48 \mathrm{~kg}$ ). Data for all three monkeys were acquired in the Research Institute of Medical Primatology, Ministry of Science and Higher Education of the Russian Federation, Sochi, Russia.

\section{Surgical procedures}

All the surgical procedures were performed under full anesthesia induced with ketamine (10 $\mathrm{mg} / \mathrm{kg}$, i.m.) and maintained under continuous intravenous infusion of propofol (1\% solution in $20 \mathrm{ml}$ Propofol $/ 20 \mathrm{ml}$ Ringer 1.8 to $6 \mathrm{ml} / \mathrm{kg} / \mathrm{h}$ ) using standard techniques. Throughout the procedures, the veterinary team continuously monitored the animal's heart rate, respiratory rate, oxygen saturation level and temperature. Surgical implantations were performed during a single operation lasting approximately 8 hours. We fixed monkeys' head in a stereotaxic frame securing the cervical spine in prone and flat position. First, we implanted two silicon cuff electrodes (Microprobes for Life Science, Gaithersburg, MD 20879, U.S.A.) on the distal ends of the superficial branch and deep branch of radial nerve that we determined via anatomical landmarks. We then inserted EMG electrodes in the Extensor Digit. Communis, the Flexor Carpi Radialis and the Flexor Digit. Superficialis. We stimulated electrically two branches of radial nerve and look at the EMG response to verify which branch was the muscle branch and which one was the cutaneous branch. Second, we implanted the brain array using a pneumatic insertion system (Blackrock Microsystem). We performed a craniotomy and we incised the dura in order to get clear access to the central sulcus. We identified motor and sensory brain areas through anatomical landmarks and intra-surgical micro-stimulation. Specifically, we verified that electrical stimulation of the motor cortex induced motor responses in the hand muscles (Extended Data Figure 1a). We then determined the position of the somatosensory area $\mathrm{S} 1$ in relation to this spot and implanted the UTAH array electrode (Blackrock Microsystems, Salt Lake City, UT, U.S.A.) across Areas 1 and 2, $1.2 \mathrm{~mm}$ lateral to midline and $3.1 \mathrm{~mm}$ deep using a pneumatic inserter (Blackrock Microsystems, Salt Lake City, UT, U.S.A.).

Finally, we performed a laminectomy from T1 to C3 vertebrae and exposed the cervical spinal cord. We implanted a 32-channel linear probe (linear Probe with Omnetics Connector 32 pins - A1x32-15mm-50-177-CM32; NeuroNexus, Ann Arbor, MI, U.S.A.) in the gray matter at the C5 spinal segment. To implant the probe, we opened the dura mater and performed a small hole in the pia using a surgical needle through which penetration of the probe with micromanipulators was possible. We implanted the arrays using MM-3 micromanipulators (Narishige, Tokyo, Japan). Experiments in all three monkeys were terminal. At the end the animals were euthanized with a single injection of penthobarbital $(60 \mathrm{mg} / \mathrm{kg})$ and perfused with PFA for further tissue processings.

\section{Electrophysiology in sedated monkeys}

Monkeys were sedated with a continuous intravenous infusion of propofol that minimizes effects on spinal cord stimulation ${ }^{51}$. Through the electrode contact of cuff electrodes we delivered single pulses of cathodic, charge balanced, symmetric square pulses (with pulse width of $0.5 \mathrm{~ms}$ ). We provided the stimulation using AM stimulators Model 2100 (A-M Systems, 
Sequim, WA, USA). Electromyographic and neural signals were acquired using the RHS recording system with 32-channel headstages (Intan Technologies, Los Angeles, CA, U.S.A.) at a sampling frequency of $30 \mathrm{kHz}$.

\section{Data analysis}

We applied all data analysis techniques offline.

\section{Pre-processing}

We filtered raw signals recorded with 32 - electrode array implanted in the spinal cord, as well as signals documented with UTAH array in somatosensory cortex with comb filter to remove artefacts on $50 \mathrm{~Hz}$ and its harmonics. We designed digital infinite impulse response filter as a group of notch filters that are evenly spaced at exactly $50 \mathrm{~Hz}$.

We detected single pulses of deep branch of radial nerve and extracted $430 \mathrm{~ms}$ of the intaspinal and intra-cortical signal post stimulation.

\section{Identification of sensory afferent volleys}

We observed afferent volleys, evoked with muscle nerve stimulation in low-frequency domain of the signal. We applied $3^{\text {rd }}$ order Butterworth digital filter and extracted the signal from $10-$ $300 \mathrm{~Hz}$. Afferent volley is defined as a first volley after the stimulation pulse. We observed stimulation artefact $0.5 \mathrm{~ms}$ after the stimulation pulse in triggered-average signal and used that as a verification that the volleys that occur 3 - $4 \mathrm{~ms}$ after the stimulation (unique physiology of a single animal causes these variations) are really afferent volleys carrying proprioceptive information. We quantified the amount of processed proprioceptive information by measuring peak-to-peak amplitude values of the afferent volleys.

We applied similar procedure to extract the afferent volleys recorded in somatosensory cortex. We present suppression of proprioceptive information caused by the concurrent stimulation of cutaneous nerve in each channel of cortex electrode as a normalized value that spans in the range from -1 to 1 . We define it as difference of peak-to-peak amplitude values in the two conditions (muscle nerve stimulation and both muscle and cutaneous nerves stimulation), normalized by the peak-to-peak amplitude value of afferent volley in muscle nerve stimulation condition.

\section{Characterization and quantification of neural spiking activity}

We extracted neural spiking activity by applying 3rd order Butterworth digital filter to the raw signal, separating the signal in frequency range from $800 \mathrm{~Hz}$ to $5000 \mathrm{~Hz}$. We detected the spikes using thresholding algorithm ${ }^{52}$. We determined the threshold value separately for each recording channel. To detect the accurate threshold value, we concatenated all data sets that we aim to analyze in a single file. All analyzed data sets were concatenated in a single file in order to detect proper threshold value. The same procedure was applied to intra-spinal and intra-cortical recordings.

Multiunit activity is presented in form of rasterplot and quantified with peri-stimulus time histrogram (PSTH). Each dot in rasterplot represents a single detected spike. Every rasteplot row corresponds to the intra-spinal or intra-cortical activity perturbed with a single muscle nerve stimulus pulse. PSTH is quantified with mean event rate, defined as the average number of spikes across all single pulses of muscle nerve stimulation, within defined time frame. 


\section{Neural manifold and trajectory length}

To project the trajectories in the neural manifold, we previously computed multi-unit firing rates for each condition. We calculated the firing rate for every $100 \mathrm{~ms}$ with a sliding window of 10 $\mathrm{ms}$. We zero-padded the first repetition for $90 \mathrm{~ms}$ and then overlapped $90 \mathrm{~ms}$ from the previous repetition for the rest of repetitions. The final step to smooth the firing rate was the application of a Gaussian kernel (s.d. $20 \mathrm{~ms}$ ) to the binned square-root-transformed firings (10 ms bin size) of each recorded multiunit. For each condition, this resulted in a matrix of dimensions $C$ $x T$, where $C$ is the number of channels in the dorso-ventral linear probe and $T$ is the number of $10 \mathrm{~ms}$ windows in a repetition concatenated for all the repetitions within a condition. Subsequently, we proceed to eliminate noisy repetitions. We discarded those repetitions within each condition whose s.d. was greater than twice the total s.d. across all repetitions plus the total mean of the s.d. across all repetitions for that condition. For cortical data, we previously converted the distribution of s.d. to a lognormal distribution to apply this outlier cleaning rule.

To calculate the latent dynamics for each monkey, we z-scored each condition's firing rate before applying dimensionality reduction principal component analysis (PCA) to the concatenated firing rates. We selected the first 3 principal components that explained most of the variance ( $\sim 65 \%$ for all three monkeys) as neural modes to define the neural manifold. In this low dimensionality space, we proceeded by eliminating repetitions as a function of the distance to the median trajectory. In particular, we computed the median trajectory for each 10 ms window for each condition. For each window, we calculated the distance between the median trajectory and the trajectory elicited by each repetition within a condition. 25th and 75th percentiles of the obtained distances allowed to discard trajectories whose distance was greater than the 75th percentile plus 1.5 times the inter-quartile range of the averaged trajectory for that repetition across all $10 \mathrm{~ms}$ windows. The same criterium was applied for the lower range. Finally, we quantified the trajectory length for the remaining repetitions for each condition and calculated the average trajectory length across all $10 \mathrm{~ms}$ windows.

\section{Statistical procedures}

Multi-group significance comparison of data obtained from the neural manifold for each condition in all three monkeys was tested using Kruskal-Wallis test. The level of significance was set at ${ }^{* * *} p<0.005$.

Significance of suppressed peak-to-peak amplitude values of afferent volleys was analyzed with one-way analysis of variance revealed (ANOVA). Each point represents the peak-to-peak amplitude as a response to a single stimulus pulse. Boxplots are show: the central mark indicates the median, and the bottom and top edges of the box indicate the 25th and 75th percentiles, respectively. The whiskers extend to the most extreme data points not considered outliers, and the outliers are plotted individually using the 'o' symbol. The level of significance was set at ${ }^{* * *} p<0.001$ and ${ }^{* *} p<0.005$. 


\section{References}

1. Raspopovic, S., Valle, G. \& Petrini, F. M. Sensory feedback for limb prostheses in amputees. Nat. Mater. 20, 925-939 (2021).

2. Raspopovic, S. et al. Restoring Natural Sensory Feedback in Real-Time Bidirectional Hand Prostheses. Sci. Transl. Med. 6, 222ra19-222ra19 (2014).

3. Formento, E. et al. Electrical spinal cord stimulation must preserve proprioception to enable locomotion in humans with spinal cord injury. Nat. Neurosci. 21, 1728-1741 (2018).

4. Hughes, C. L. et al. Perception of microstimulation frequency in human somatosensory cortex. eLife 10, e65128 (2021).

5. Lee, B. et al. Engineering Artificial Somatosensation Through Cortical Stimulation in Humans. Front. Syst. Neurosci. 12, 24 (2018).

6. Rognini, G. et al. Multisensory bionic limb to achieve prosthesis embodiment and reduce distorted phantom limb perceptions. J. Neurol. Neurosurg. Psychiatry 90, 833-836 (2019).

7. Tan, D. W. et al. A neural interface provides long-term stable natural touch perception. Sci. Transl. Med. 6, 257ra138-257ra138 (2014).

8. Davis, T. S. et al. Restoring motor control and sensory feedback in people with upper extremity amputations using arrays of 96 microelectrodes implanted in the median and ulnar nerves. $J$. Neural Eng. 13, 036001 (2016).

9. Petrini, F. M. et al. Sensory feedback restoration in leg amputees improves walking speed, metabolic cost and phantom pain. Nat. Med. 25, 1356-1363 (2019).

10. Navarro, X. et al. A critical review of interfaces with the peripheral nervous system for the control of neuroprostheses and hybrid bionic systems. J. Peripher. Nerv. Syst. 10, 229-258 (2005).

11. Petrini, F. M. et al. Enhancing functional abilities and cognitive integration of the lower limb prosthesis. Sci. Transl. Med. 11, eaav8939 (2019).

12. Valle, G. et al. Mechanisms of neuro-robotic prosthesis operation in leg amputees. Sci. Adv. 7, eabd8354 (2021). 
13. D’Anna, E. et al. A somatotopic bidirectional hand prosthesis with transcutaneous electrical nerve stimulation based sensory feedback. Sci. Rep. 7, 10930 (2017).

14. Chandrasekaran, S. et al. Sensory restoration by epidural stimulation of dorsal spinal cord in upperlimb amputees. http://medrxiv.org/lookup/doi/10.1101/19009811 (2019) doi:10.1101/19009811.

15. Ortiz-Catalan, M., Mastinu, E., Sassu, P., Aszmann, O. \& Brånemark, R. Self-Contained Neuromusculoskeletal Arm Prostheses. N. Engl. J. Med. 382, 1732-1738 (2020).

16. Zollo, L. et al. Restoring Tactile sensations via neural interfaces for real-time force-and-slippage closed-loop control of bionic hands. Sci. Robot. 4, eaau9924 (2019).

17. Graczyk, E. L., Delhaye, B. P., Schiefer, M. A., Bensmaia, S. J. \& Tyler, D. J. Sensory adaptation to electrical stimulation of the somatosensory nerves. J. Neural Eng. 15, 046002 (2018).

18. Strauss, I. et al. Characterization of multi-channel intraneural stimulation in transradial amputees. Sci. Rep. 9, 19258 (2019).

19. Petrini, F. M. et al. Six-Month Assessment of a Hand Prosthesis with Intraneural Tactile Feedback. Ann. Neurol. 85, 137-154 (2019).

20. Dhillon, G. S., Lawrence, S. M., Hutchinson, D. T. \& Horch, K. W. Residual function in peripheral nerve stumps of amputees: implications for neural control of artificial limbs. J. Hand Surg. 29, 605615; discussion 616-618 (2004).

21. Horch, K., Meek, S., Taylor, T. G. \& Hutchinson, D. T. Object discrimination with an artificial hand using electrical stimulation of peripheral tactile and proprioceptive pathways with intrafascicular electrodes. IEEE Trans. Neural Syst. Rehabil. Eng. Publ. IEEE Eng. Med. Biol. Soc. 19, 483-489 (2011).

22. Saal, H. P. \& Bensmaia, S. J. Biomimetic approaches to bionic touch through a peripheral nerve interface. Neuropsychologia 79, 344-353 (2015).

23. Overstreet, C. K., Cheng, J. \& Keefer, E. W. Fascicle specific targeting for selective peripheral nerve stimulation. J. Neural Eng. 16, 066040 (2019). 
24. George, J. A. et al. Biomimetic sensory feedback through peripheral nerve stimulation improves dexterous use of a bionic hand. Sci. Robot. 4, eaax2352 (2019).

25. Wendelken, S. et al. Restoration of motor control and proprioceptive and cutaneous sensation in humans with prior upper-limb amputation via multiple Utah Slanted Electrode Arrays (USEAs) implanted in residual peripheral arm nerves. J. NeuroEngineering Rehabil. 14, 121 (2017).

26. Charkhkar, H. et al. High-density peripheral nerve cuffs restore natural sensation to individuals with lower-limb amputations. J. Neural Eng. 15, 056002 (2018).

27. Rattay, F. Analysis of Models for External Stimulation of Axons. IEEE Trans. Biomed. Eng. BME-33, 974-977 (1986).

28. Capogrosso, M. et al. A Computational Model for Epidural Electrical Stimulation of Spinal Sensorimotor Circuits. J. Neurosci. 33, 19326-19340 (2013).

29. Schiefer, M. A., Triolo, R. J. \& Tyler, D. J. A Model of Selective Activation of the Femoral Nerve With a Flat Interface Nerve Electrode for a Lower Extremity Neuroprosthesis. IEEE Trans. Neural Syst. Rehabil. Eng. 16, 195-204 (2008).

30. McNeal, D. R. Analysis of a Model for Excitation of Myelinated Nerve. IEEE Trans. Biomed. Eng. BME-23, 329-337 (1976).

31. Raspopovic, S., Capogrosso, M. \& Micera, S. A Computational Model for the Stimulation of Rat Sciatic Nerve Using a Transverse Intrafascicular Multichannel Electrode. IEEE Trans. Neural Syst. Rehabil. Eng. 19, 333-344 (2011).

32. Greiner, N. et al. Recruitment of upper-limb motoneurons with epidural electrical stimulation of the cervical spinal cord. Nat. Commun. 12, 435 (2021).

33. Raspopovic, S., Capogrosso, M., Badia, J., Navarro, X. \& Micera, S. Experimental Validation of a Hybrid Computational Model for Selective Stimulation Using Transverse Intrafascicular Multichannel Electrodes. IEEE Trans. Neural Syst. Rehabil. Eng. 20, 395-404 (2012). 
34. Kibleur, P. et al. Spatiotemporal Maps of Proprioceptive Inputs to the Cervical Spinal Cord During Three-Dimensional Reaching and Grasping. IEEE Trans. Neural Syst. Rehabil. Eng. 28, 1668-1677 (2020).

35. Kandel, E., Schwartz, J. \& Jessel, T. Principles of Neuro Science. (The McGraw-Hill Companies, 2000).

36. Rudomin, P. \& Schmidt, R. F. Presynaptic inhibition in the vertebrate spinal cord revisited. Exp. Brain Res. 129, 1-37 (1999).

37. Confais, J., Kim, G., Tomatsu, S., Takei, T. \& Seki, K. Nerve-Specific Input Modulation to Spinal Neurons during a Motor Task in the Monkey. J. Neurosci. 37, 2612-2626 (2017).

38. Seki, K., Perlmutter, S. I. \& Fetz, E. E. Sensory input to primate spinal cord is presynaptically inhibited during voluntary movement. Nat. Neurosci. 6, 1309-1316 (2003).

39. Gallego, J. A., Perich, M. G., Miller, L. E. \& Solla, S. A. Neural Manifolds for the Control of Movement. Neuron 94, 978-984 (2017).

40. Gallego, J. A., Perich, M. G., Miller, L. E. \& Solla, S. A. Neural Manifolds for the Control of Movement. Neuron 94, 978-984 (2017).

41. Gallego, J. A., Perich, M. G., Chowdhury, R. H., Solla, S. A. \& Miller, L. E. Long-term stability of cortical population dynamics underlying consistent behavior. Nat. Neurosci. 23, 260-270 (2020).

42. Trautmann, E. M. et al. Accurate Estimation of Neural Population Dynamics without Spike Sorting. Neuron 103, 292-308.e4 (2019).

43. Perich, M. G. et al. Motor cortical dynamics are shaped by multiple distinct subspaces during naturalistic behavior. http://biorxiv.org/lookup/doi/10.1101/2020.07.30.228767 doi:10.1101/2020.07.30.228767.

44. Abraira, V. E. \& Ginty, D. D. The sensory neurons of touch. Neuron 79, 618-639 (2013).

45. Balaguer, J.-M. \& Capogrosso, M. A Computational Model of the Interaction Between Residual Cortico-Spinal Inputs and Spinal Cord Stimulation After Paralysis. in 2021 10th International 
IEEE/EMBS Conference on Neural Engineering (NER) 251-254 (IEEE, 2021). doi:10.1109/NER49283.2021.9441219.

46. Formento, E., D’Anna, E., Gribi, S., Lacour, S. P. \& Micera, S. A biomimetic electrical stimulation strategy to induce asynchronous stochastic neural activity. J. Neural Eng. 17, 046019 (2020).

47. Versteeg, C., Chowdhury, R. H. \& Miller, L. E. Cuneate nucleus: The somatosensory gateway to the brain. Curr. Opin. Physiol. 20, 206-215 (2021).

48. London, B. M. \& Miller, L. E. Responses of somatosensory area 2 neurons to actively and passively generated limb movements. J. Neurophysiol. 109, 1505-1513 (2013).

49. Bensmaia, S. J. Biological and bionic hands: natural neural coding and artificial perception. Philos Trans R Soc Lond B Biol Sci 370, 20140209 (2015).

50. Page, D. M. et al. Discriminability of multiple cutaneous and proprioceptive hand percepts evoked by intraneural stimulation with Utah slanted electrode arrays in human amputees. $J$. NeuroEngineering Rehabil. 18, 12 (2021).

\section{Methods only references}

51. Toossi, A. et al. Effect of anesthesia on motor responses evoked by spinal neural prostheses during intraoperative procedures. J. Neural Eng. 16, 036003 (2019).

52. Quiroga, R. Q., Nadasdy, Z. \& Ben-Shaul, Y. Unsupervised Spike Detection and Sorting with Wavelets and Superparamagnetic Clustering. Neural Comput. 16, 1661-1687 (2004). 